\title{
Assessment of Stress Distribution Using Equator Attachment Design in Mandibular Implant-retained Overdentures in Comparison with Ball and Socket Attachment Design: 3D Finite Element Analysis
}

\author{
Mahmoud Samir ${ }^{1 *}$, Hamdi Abo Alfotouh ${ }^{2}$ and Mostafa Helmy \\ ${ }^{1}$ MSc Student of Master of Implantology Program, Faculty of Dentistry, Cairo \\ University, Cairo, Egypt \\ ${ }^{2}$ Professor of Prosthodontics Department, Faculty of Dentistry, Cairo University, \\ Cairo, Egypt \\ ${ }^{3}$ Associate Professor of Prosthodontics Department, Faculty of Dentistry, Cairo \\ University ,Cairo, Egypt \\ *Corresponding Author: Mahmoud Samir, MSc Student of Master of Implantology \\ Program, Faculty of Dentistry, Cairo University, Cairo, Egypt.
}

Received: November 13, 2020

Published: December 16, 2020

(C) All rights are reserved by Mahmoud

Samir., et al.

\begin{abstract}
Aim: To accomplish a comparative study between two types of attachment Equator and Ball/Socket in mandibular implant supported overdenture by using finite element analysis and assessment of stress distribution on the bone, implant. overdenture, mucosa and attachment.

Methodology: Three-dimensional models simulated a clinical situation where an edentulous mandible was restored by two different treatment options using implant overdentures. The overdentures were supported by two implants placed at the approximate canine region using Ball/Socket and Equator attachments. The load was applied of value of 50,100,200 $\mathrm{N}$ with angle of vertical $90^{\circ}$ then the oblique load with angel $45^{\circ}$.

Results: Attachment total deformation with using ball and socket attachments deforms much more than using equator attachment. Similarly, attachments Von Mises stress increase dramatically with ball and socket attachment. Central incisor loading did not show as much difference as molar area loading.

Conclusion: Equators body and its caps (flexible and metallic) received lower stresses that indicating longer life time and longer periods between successive maintenances.
\end{abstract}

Keywords: Bone; Ball; Socket

\section{Introduction}

A lot of people around the world lost their teeth because of caries, periodontal disease, accidents and oral cancer. They are classified either partial or completely edentulous patients.

The edentulous patients are facing many problem as the reduced number of teeth makes the chewing and mastication is more difficult which affect their normal health. The patients try to avoid the hard and tough food as they are very difficult to chew.
Edentulous patients are considered, according to the world health organization, disabled and handicapped because they are not able to masticate and speak well [1]. Edentulism is a worldwide phenomenon. The rate of edentulism increases with age.

The latest researches show that there are no gender biases for edentulous patients, both men and women nearly equal. The most popular and traditional treatment for edentulous patient is the complete denture. 
The complete denture has a lot complications that may be local or systemic such as loss of complete denture retention and its stability, accelerated jaw bone loss, gingival hyperplasia, denture stomatitis, inconvenient, dull the senses in the mouth, repeatedly lose their fit, become more loose over time and it only provides a small fraction of the chewing power of natural teeth [2].

The advent of dental implant, over the last two decades, has a great effect for prosthetic management of completely edentulous patients. Per-Ingvar Brånemark, Swedish surgeon, discovered the implant in 1960's. Dental implants may be made of titanium, titanium alloy or zirconia. Zirconia implant is the most recent invention. All these materials are biocompatible with human body and have no adverse effects. It compensates for the function, esthetic requirements and needs minimal maintenance [3].

Root supported overdentures use the remaining root structure to provide stability, retention and support for the overdenture. The existence of periodontal ligaments preserve alveolar ridge height, width, shape and decrease the natural bone loss that occurs after tooth extraction.

Dental implant attachment systems nowadays are classified into two main groups: bar and stud attachments.

The selected attachments for implant supported overdentures should have enough retentive properties to enhance the stability of the restoration. The way stress is applied to implants after osseo-integration was one of the important factors considered and studied in implant dentistry.

The distribution of forces in peri-implant bone has been investigated by finite element analyses in several studies. Recently, stress distribution in bone correlated with implant-supported prosthesis design has been investigated primarily by means of two-dimensional (2D) and three-dimensional (3D) finite element analyses (FEAs). Cases comparing the accuracy of these analyses found that, if detailed stress information is essential, then three dimensional modeling is mandatory [4].

Many different attachments available today may be used to retain implant-retained overdentures. However, the selected attachment used in implant-retained overdenture has a potential effect on implant survival rate, marginal bone loss, soft tissue complications, retention, stress distribution, maintenance complications and patient's satisfaction [5].
(El-Anwar., et al. 2015) studied the pattern of stress distribution of ball \socket attachment system. The result was highly stressed abutment neck and less stresses received on mucosa and cortical bone. The conclusion was that the use of ball \socket attachment system is preferable for weak bone, but it has less prosthetic lifetime in comparison to other separate attachments types [6].

(Weerapan Aunmeungtong., et al. 2017) stated that there was a significant difference in marginal bone loss and patient satisfaction between those receiving mini dental implants with Equator attachments and conventional dental implants with ball attachments [7].

(Marcello-Machadoe., et al. 2018) concluded that NDI connected to locking taper Equator attachments showed a stable clinical behavior as an MO retainer for edentulous patients with clinical mandibular atrophy [8].

The null hypothesis of this study assumed that that there will be no difference between equator attachment design Implant retained overdenture restoration using two implants in stress distribution level at the implant/bone interface compared to Ball and Socket attachment design Implant retained overdenture

\section{Materials and Methods}

The 3D FEA study resembles a clinical situation where completely edentulous mandible restored with two implants retained overdenture. The overdenture was supported by two implants in the canine regions. Perfect osseo-integration was assumed to be presented between implants and bone. That a finite element model was prepared based on Geng., et al. [9], and El-Anwar., et al. [10,11]. Two attachment systems were studied and compared; Equator and ball and socket, different load cases were tested to find out the better design for bone stresses and deformations.

The 3D FE model components as the overdenture, mucosa, implant, equators, ball and socket' attachment, metallic caps, rubber caps, cortical and cancellous bones were created in "Autodesk Inventor" Version 8 (Autodesk Inc., San Rafael, CA, USA), then exported as SAT files. These components were assembled in ANSYS environment (ANSYS Inc., Canonsburg, PA, USA). Where the designs of the implant and the attachment systems were taken from the manufacturer data. The system analyzed in this investigation consisted of the commonly available root form threaded titanium dental implant (Zimmer Dental Inc, USA). Ball and socket (Zimmer Dental Inc, USA) and OT Equator (MIS Implants Technologies Ltd,

Citation: Mahmoud Samir., et al. "Assessment of Stress Distribution Using Equator Attachment Design in Mandibular Implant-retained Overdentures in Comparison with Ball and Socket Attachment Design: 3D Finite Element Analysis". Acta Scientific Dental Sciences 5.1 (2021): 63-70. 
Assessment of Stress Distribution Using Equator Attachment Design in Mandibular Implant-retained Overdentures in Comparison with Ball and Socket Attachment Design: 3D Finite Element Analysis

dimensions were taken from manufacturer catalogues. The root form dental implant had a diameter of $4.1 \mathrm{~mm}$ (Model TSVM Implant) with $3.5 \mathrm{~mm}$ Apex Diameter, a length of $10 \mathrm{~mm}$ with internal connection.

All parts of the mandible as assembled appeared on Inventor screen. All these parts in addition to the implant, abutment, and magnetic attachment were exported from Inventor as SAT files [5]. Then set of Boolean operations were carried out to assemble all the model components before meshing.

The simulated peri-implant bone included an inner layer representing cancellous bone of $22 \mathrm{~mm}$ height and $14 \mathrm{~mm}$ width covered by an outer thin layer of cortical bone of $2 \mathrm{~mm}$ thickness. The simulated covering mucosal layer was of $2 \mathrm{~mm}$ thickness. The prosthetic acrylic overdenture was simulated of height $8 \mathrm{~mm}$ and width of 8.8 $\mathrm{mm}[12,13]$.

All materials used in this study were assumed to be isotropic, homogenous, and linearly elastic and its properties are listed in table 1 [14].

\begin{tabular}{|l|c|c|}
\hline Material & Young's Modulus [MPa] & $\begin{array}{c}\text { Poisson's } \\
\text { Ratio }\end{array}$ \\
\hline Cortical & 13,700 & 0.30 \\
\hline Cancellous & 1,370 & 0.30 \\
\hline Implant - attachment & $\begin{array}{c}110,000 \\
\text { (Per ASTM E8-04) }\end{array}$ & 0.33 \\
\hline Metallic cap & $\begin{array}{c}110,000 \\
\text { (Per ASTM E8-04) }\end{array}$ & 0.33 \\
\hline Nylon ring & 350 & 0.40 \\
\hline Mucosa & 10 & 0.40 \\
\hline Overdenture & 2,700 & 0.35 \\
\hline
\end{tabular}

Table 1: Mechanical properties of materials used in the finite element model.

Set of Boolean operations between the modeled components were performed before obtaining the complete model(s) assembled. The meshing of these components was done by 3D solid element (SOLID187) which has three degrees of freedom (translation in main axes directions) [15]. The resulted numbers of nodes and elements are listed in table 2.

Each model was subjected to four loading conditions of $100 \mathrm{~N}$, $50 \mathrm{~N}$ vertically and oblique (antroposterior) at central incisors, respectively. In addition to $200 \mathrm{~N}, 100 \mathrm{~N}$ unilaterally vertically and oblique (bucclingual) at molar region, respectively. The lowest plane of the model was considered fixed in the three directions as a boundary condition.

\begin{tabular}{|l|c|c|c|c|}
\hline \multirow{2}{*}{} & \multicolumn{2}{|c|}{ Equator Model } & \multicolumn{2}{c|}{ Ball and socket Model } \\
\cline { 2 - 5 } & Nodes & Elements & Nodes & Elements \\
\hline Cortical Bone & 22,844 & 11,500 & 21,226 & 9,334 \\
\hline Spongy Bone & 53,115 & 31,171 & 54,379 & 34,949 \\
\hline 2 x Implant & 43,633 & 24,816 & 42,987 & 24,753 \\
\hline 2 x Attachment & 10,299 & 5,855 & 10,423 & 6,012 \\
\hline 2 x Rubber Ring & 1,510 & 755 & 1,630 & 18,430 \\
\hline 2 x Metal Cap & 1,128 & 516 & 1,100 & 3,316 \\
\hline Mucosa & 17,957 & 3,342 & 16,313 & 6,887 \\
\hline Overdenture & 10,051 & 5,458 & 10,648 & 15,262 \\
\hline
\end{tabular}

Table 2: Number of nodes and elements in all meshed components.

\section{Results}

obtained results were demonstrated and compared. In each run (case study) each model components results were taken as screen shots from ANSYS. The definition of most important results obtained and demonstrated below as follows;

- $\quad$ Maximum principal (tensile) stress

- Minimum principal (compressive) stress

- $\quad$ Maximum shear Stress

- $\quad$ Von Mises (Equivalent) stress

- Total deformation

- Deformation in Z direction (vertical)

- Deformation in Y direction (Antropostrior)

- Deformation in X direction (Buccolingual).

For convenience total deformation (Usum), and Von Mesis Stress (Equivalent) (Svon) will be presented. That will give sufficient information about each component behavior of the studied system.

All components (Cortical Bone- Spongy Bone- Implant- Attachments) showed safe level of stresses, that no worry from failure of any part of the model, because the resultant stresses do not exceed the physiological limits.

Although all values of deformations and stresses appeared on both attachments were within physiological limits. According to comparison presented in Figure 1 the attachment total deformation with using ball and socket attachments deforms much more than using equator attachment. Similarly, attachments Von Mises stress increase dramatically with ball and socket attachment. Central incisor loading did not show as much difference as molar area loading. 


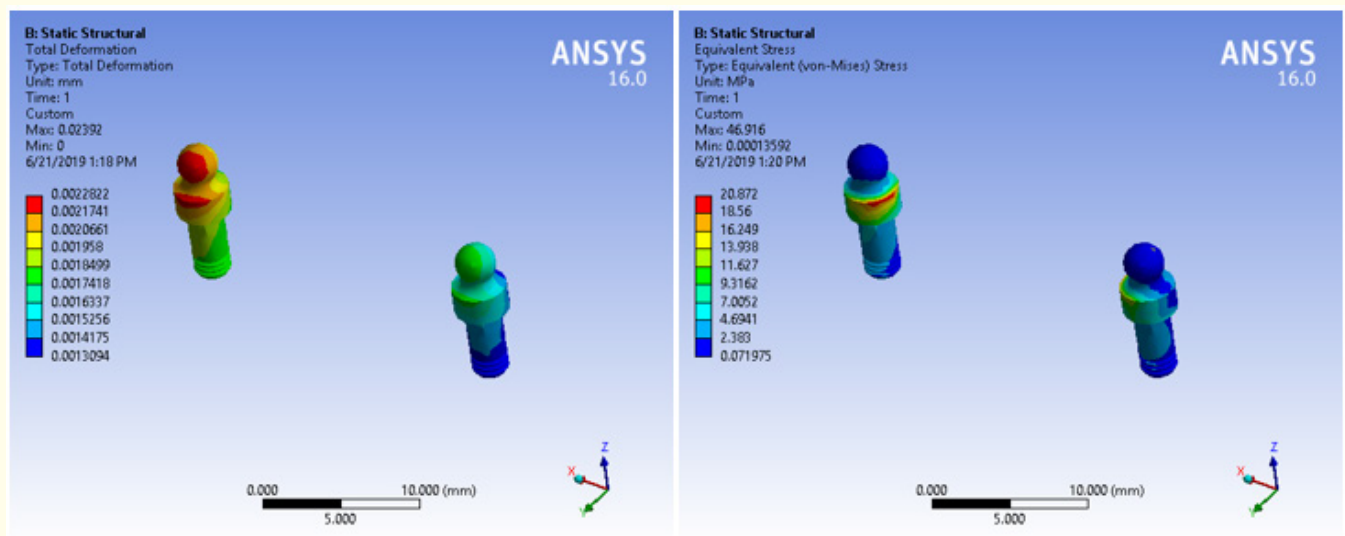

Figure 1: Equator results, vertical $100 \mathrm{~N}$ on central incisor.
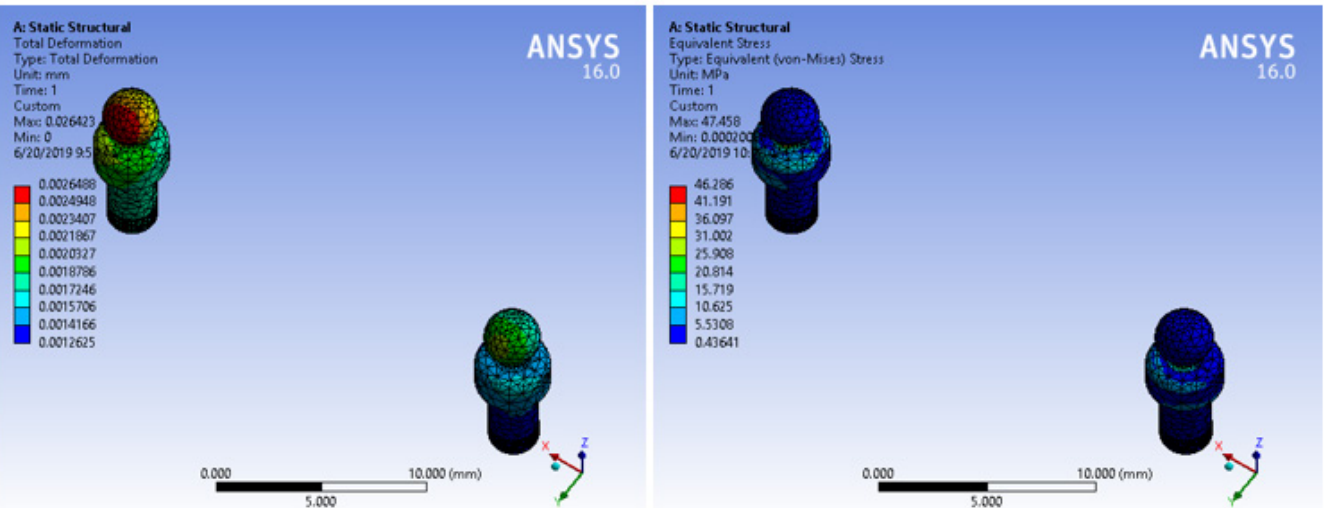

Figure 2: Ball and Socket results, vertical $100 \mathrm{~N}$ on central incisor.
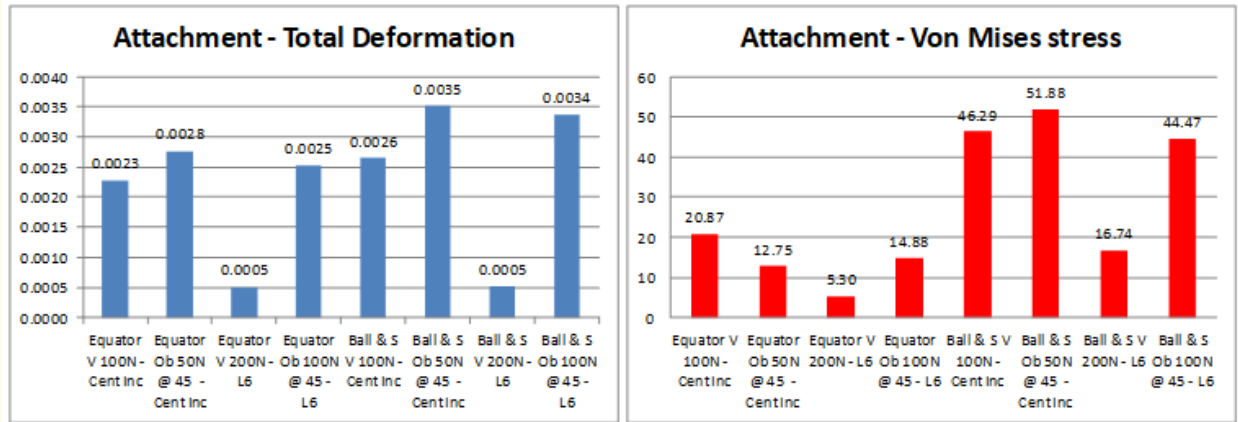

Figure 3: Attachment results comparisons. 
Cortical bone results showed that the cortical bone total deformation was not sensitive to attachment type. On the other hand, cortical bone Von Mises stress increase dramatically (about 30\%) with equator attachments when compared to ball and socket attachment. Central incisor loading showed higher stresses than loading in molar zone.

Spongy bone total deformation and stresses with using Equator attachments are slightly less than using ball and socket attachment.
As noticed on cortical bone, central incisor loading showed more stresses than molar area loading. On the other hand, the attachment total deformation with using ball and socket attachments deforms much more than using equator attachment. Similarly, attachments Von Mises stress increase dramatically with ball and socket attachment. Central incisor loading did not show as much difference as molar area loading.
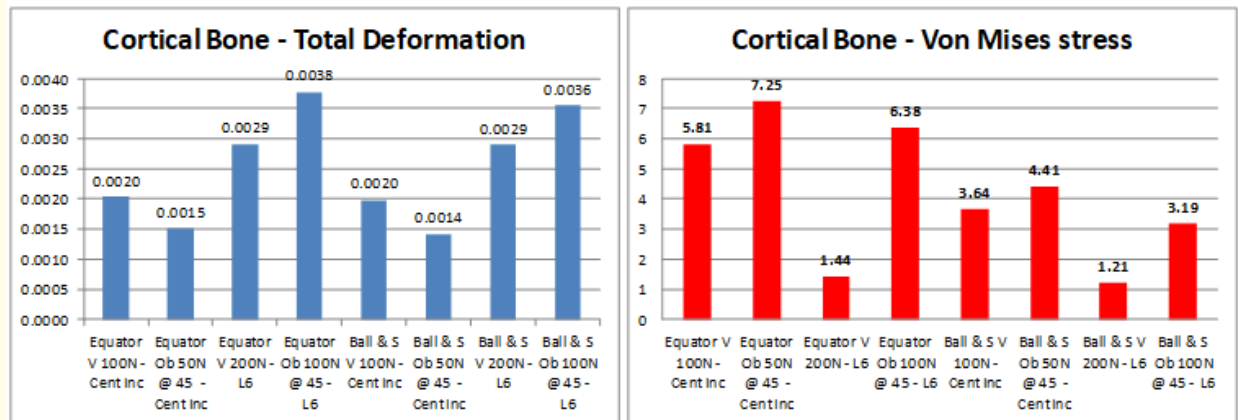

Figure 4: Cortical bone results comparisons.
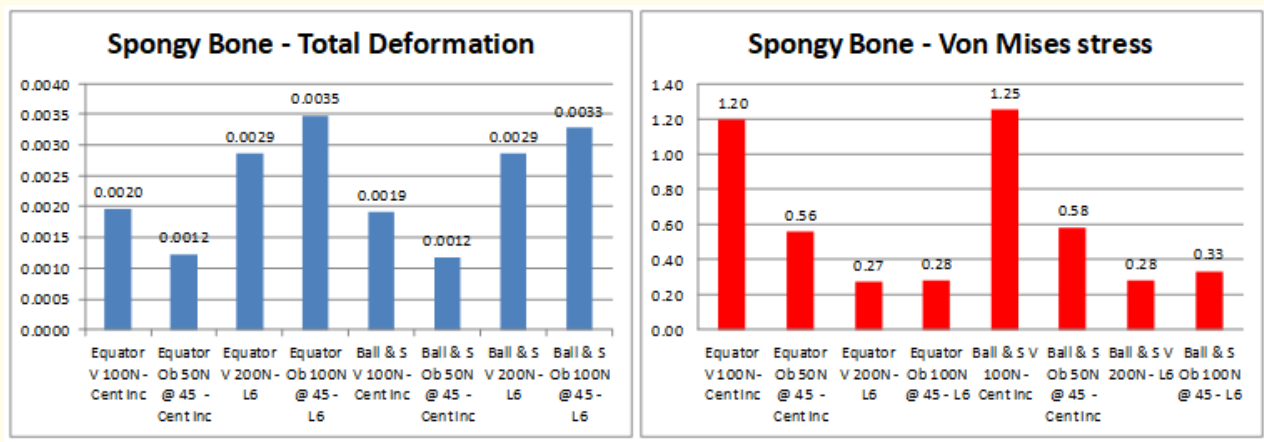

Figure 5: Spongy bone results comparisons.

On both caps (flexible and metallic) the oblique loading showed higher deformations and stresses than vertical one on caps. While equators metal caps in general showed lower deformation and stresses. The overdenture total deformation with using ball and socket attachments deforms less than using equator attachment. On the other hand, overdenture Von Mises stress seem to be equivalent for both attachments. The mucosa total deformation with using ball and socket attachments deforms much more than using equators attachment.

Implant results: Implant total deformation with using ball and socket attachments deforms within the same order of equator attachment. While, implants Von Mises stress slightly increase with equator attachment. Central incisor loading did not show as much difference as molar area loading. 

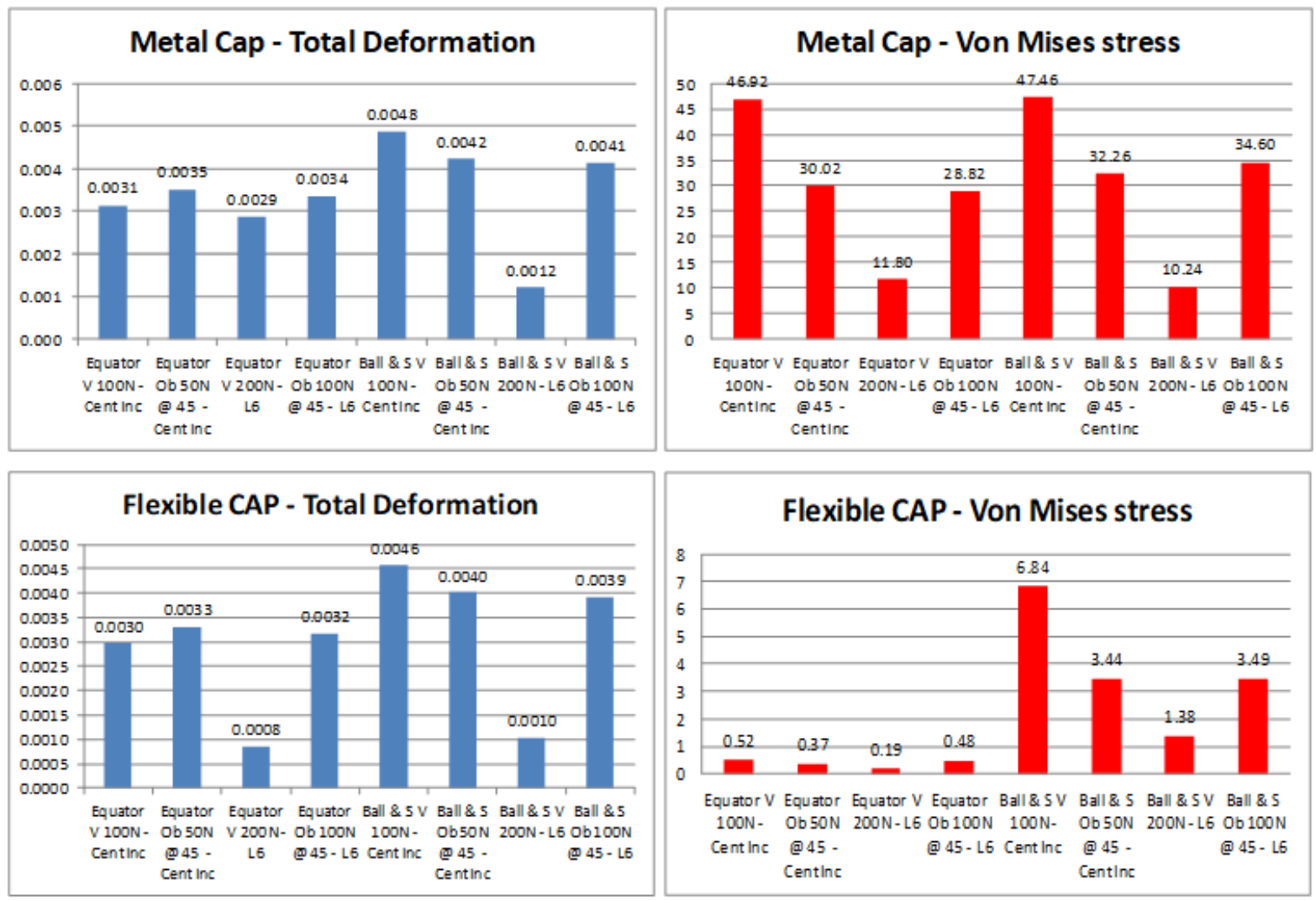

Figure 6: Metal and flexible cap comparisons.
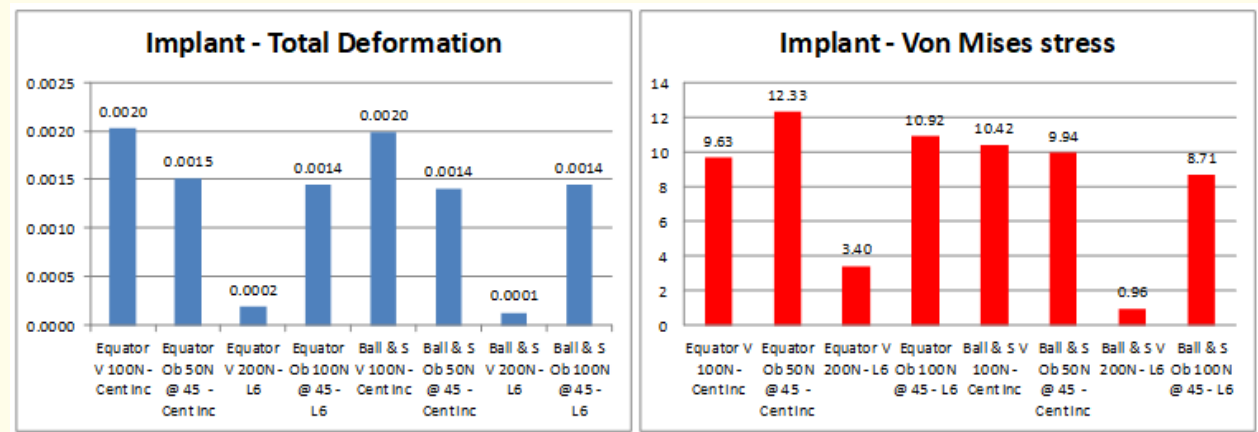

Figure 7: Implant result comparisons.

\section{Discussion}

The finite element analysis is a reliable technique for analyzing stresses around the dental implant, bone and attachment systems. In this in-vitro study, the authors aimed to assess applied stresses on the implants comparing between equator attachment systems and ball \&socket attachment systems in mandibular implant-retained overdenture.

In-vitro study was shot in this research as the attachments are recently launched in the markets. Thus, it is preferred to investi- 
gate this new attachment system outside the patient's mouth for better understanding of its effect on the bone stresses and deformations and other finite element model components. In addition, in-vitro study can be done with less ethical and safety concern.

The load applied on central incisor with magnitude of $100 \mathrm{~N}$ in a vertical $90^{\circ}$ direction, and $50 \mathrm{~N}$ in oblique $45^{\circ}$ bucco- lingual direction.

The applied load on molar region was unilateral of $200 \mathrm{~N}, 100 \mathrm{~N}$ in a vertical $90^{\circ}$ and in oblique $45^{\circ}$ bucco-lingual directions respectively. The choice of a load direction was determined since the implant overdenture in an edentulous patient is subjected to a vertical biting force perpendicular to the occlusal plane and an oblique force applied on the buccal surface to represent the chewing forces. In addition, the choice of a load of $200 \mathrm{~N}$ was determined by the suggested average maximum occlusal force in complete denture patients. The load was applied on the region of premolar/molar as the occlusal surface of the distal half of the second premolar and the mesial of the first molar are considered the center of mastication where eighty percent of the force of mastication falls $[16,17]$.

Under molar loading(s), the implant complex showed higher total deformation with using ball and socket attachments in comparison to equator attachment. Similarly, Von Mises stress increase dramatically with ball and socket attachment. While central incisor loading did not show as much difference as molar area loading. In other words, implants might be relaxed under ball and socket due to dissipation of most of load energy in ball attachment deformation and stresses.

Although the central incisor loading did not show as much difference as molar area loading. That may be referred to the small distance between loading site and supporting implant in case of central incisor in comparison to molar loading.

Generally, equator attachment showed superior behavior over traditional attachment (ball and socket) when loaded at central incisor, that the applied load energy mostly absorbed by implant complex.

Shorter neck of equator, may give slight advantage from the overdenture point of view, that may referred to thicker overdenture above it in comparison to ball and socket.

The ball and socket attachment showed better behavior on cortical bone and mucosa stresses only, that may be referred to receiv- ing (absorbing) load energy in deforming and stressing the ball attachment neck. Thus, less energy will be transferred to underneath structures (mucosa and cortical bone). Therefore, the null hypothesis was rejected.

These findings are similar to previous studies [6-8] which concluded that the stresses applied to equator attachment design in mandibular implant-retained overdentures and ball and socket attachment design l were within the physiological limits so it is considered as a viable treatment option.

\section{Conclusion}

All values of deformations and stresses appeared on all models' components (cortical, spongy bone, implant, attachments, caps, mucosa, and overdenture) were within physiological limits under all cases of load application.

Equator attachment showed superior behavior over traditional attachment (ball and socket) when loaded at central incisor.

Equators body and its caps (flexible and metallic) received lower stresses that indicating longer life time and longer periods between successive maintenances.

The ball and socket showed better behavior on cortical bone and mucosa stresses only.

\section{Conflict of Interest}

The authors declare no conflict of interest.

\section{Bibliography}

1. Emami Elham., et al. "The impact of edentulism on oral and general health". International Journal of Dentistry 2013 (2013): 498305.

2. Bilhan Hakan., et al. "Complication rates and patient satisfaction with removable dentures". The Journal of Advanced Prosthodontics 4.2 (2012): 109-115.

3. Buser D., et al. "Modern implant dentistry based on osseointegration: 50 years of progress, current trends and open questions". Periodontology 73.1 (2017): 7-21.

4. $\quad$ Ding Xi., et al. "Implant-bone interface stress distribution in immediately loaded implants of different diameters: a threedimensional finite element analysis". Journal of Prosthodontics: Implant, Esthetic and Reconstructive Dentistry 18.5 (2009): 393-402. 
5. F Wang., et al. "Maxillary Four Implant-retained Overdentures via Locator ${ }^{\circledR}$ Attachment: Intermediate-term Results from a Retrospective Study". Clinical Implant Dentistry and Related Research 18.3 (2016): 571-579.

6. El-Anwar MI., et al. "A Finite element study on stresses distribution of two different attachment designs under implant supported overdenture". The Saudi Dental Journal 27.4 (2015): 201-217.

7. Aunmeungtong Weerapan., et al. "Comparative clinical study of conventional dental implants and mini dental implants for mandibular overdentures: a randomized clinical trial". Clinical Implant Dentistry and Related Research 19.2 (2017): 328-340.

8. Marcello-Machado., et al. "One-year clinical outcomes of locking taper Equator attachments retaining mandibular overdentures to narrow diameter implants". Clinical Implant Dentistry and Related Research 20.4 (2018): 483-492.

9. Ozan and S Ramoglu. "Effect of Implant Height Differences on Different Attachment Types and Peri-Implant Bone in Mandibular Two-Implant Overdentures: 3D Finite Element Study". Journal of Oral Implantology 41.3 (2015): 50-59.

10. Geng Jianping., et al. "Application of the finite element method in implant dentistry". Springer Science and Business Media (2008).

11. El-Anwar MI., et al. "Influence of Number of Implants and Attachment Type on Stress Distribution in Mandibular Implant-Retained Overdentures: Finite Element Analysis". Open Access Macedonian Journal of Medical Sciences 5.2 (2017): 244-249.

12. MI El-Anwar. "Simple Technique to Build Complex 3D Solid Models". Proceeding of 19th International Conference on Computer Theory and Applications (ICCTA 2009), 17-19 Oct. Alexandria, Egypt (2009).

13. Huang HL., et al. "Comparison of implant body designs and threaded designs of dental implants: A 3-dimensional finite element analysis". International Journal of Oral and Maxillofacial Implants 22.4 (2007): 551-562.

14. Esteban Pérez-Pevida., et al. "Biomechanical Consequences of the Elastic Properties of Dental Implant Alloys on the Supporting Bone: Finite Element Analysis". BioMed Research International (2016): 9.

15. Lui J., et al. "Influence of implant number on the biomechanical behavior of mandibular implant-retained/supported overdentures: a three-dimensional finite element analysis". Journal of Dentistry 41.3 (2013): 241-249.
16. Visser Anita., et al. "Mandibular overdentures supported by two or four endosseous implants: A 5-year prospective study". Clinical Oral Implants Research 16.1 (2005): 19-25.

17. Schwitalla A D., et al. "Finite element analysis of the biomechanical effects of PEEK dental implants on the peri-implant bone". Journal of Biomechanics 48.1 (2015): 1-7.

\section{Assets from publication with us}

- Prompt Acknowledgement after receiving the article

- Thorough Double blinded peer review

- Rapid Publication

- Issue of Publication Certificate

- High visibility of your Published work

Website: $\underline{w w w}$.actascientific.com/

Submit Article: www.actascientific.com/submission.php

Email us: editor@actascientific.com

Contact us: +919182824667 